\title{
Osteoblast-induced EGFR/ERBB2 signaling in androgen-sensitive prostate carcinoma cells characterized by multiplex kinase activity profiling
}

\author{
Åse Bratland · Piet J. Boender · Hanne K. Høifødt • Ingrid H. G. Østensen · \\ Rob Ruijtenbeek · Meng-yu Wang · Jens P. Berg • Wolfgang Lilleby • \\ Øystein Fodstad · Anne Hansen Ree
}

Received: 4 June 2008/Accepted: 27 February 2009/Published online: 18 March 2009

(c) The Author(s) 2009. This article is published with open access at Springerlink.com

\begin{abstract}
Bone metastases in prostate cancer are predominantly osteoblastic. To study regulatory mechanisms underlying the establishment of prostate cancer within an osteoblastic microenvironment, human androgen-sensitive prostate carcinoma cells (LNCaP) were treated with culture medium conditioned by human osteoblast-derived sarcoma
\end{abstract}

\footnotetext{
Å. Bratland · H. K. Høifødt · M.-y. Wang · Ø. Fodstad .

A. H. Ree $(\bowtie)$

Department of Tumor Biology, Oslo University Hospital,

Montebello, 0310 Oslo, Norway

e-mail: a.h.ree@medisin.uio.no

\begin{abstract}
Å. Bratland · W. Lilleby
Division of Cancer Medicine and Radiotherapy,
\end{abstract}

Oslo University Hospital, Oslo, Norway

P. J. Boender · R. Ruijtenbeek

PamGene International BV, 's-Hertogenbosch, The Netherlands

I. H. G. Østensen

Norwegian Microarray Consortium, Oslo University Hospital,

Oslo, Norway
}

I. H. G. Østensen

Department of Molecular Biosciences, University of Oslo,

Oslo, Norway

J. P. Berg

Faculty Division Ullevål University Hospital,

University of Oslo, Oslo, Norway

Å. Bratland · Ø. Fodstad

Faculty Division The Norwegian Radium Hospital,

University of Oslo, Oslo, Norway

A. H. Ree

Faculty Division Akershus University Hospital,

University of Oslo, Oslo, Norway cells (OHS), and activated signalling pathways in the carcinoma cells were analyzed using microarrays with tyrosine kinase substrates. Network interaction analysis of substrates with significantly increased phosphorylation levels revealed that signalling pathways mediated by EGFR and ERBB2 were activated in LNCaP cells under OHS influence but also by androgen treatment. Activation of EGFR/ERBB2 signalling was also found in LNCaP cells in cocultures with OHS cells or osteoblastic cells that had been differentiated from human mesenchymal stem cells. Our experimental data suggests osteoblast-directed induction of signalling activity via EGFR and ERBB2 in prostate carcinoma cells and may provide a rationale for the use of EGFR or ERBB2 inhibition in systemic prevention or treatment of metastatic prostate cancer in the androgensensitive stage of the disease.

Keywords Androgen - EGFR - ERBB2 - Kinase array · Metastasis · Osteoblast · Prostate carcinoma

\author{
Abbreviations \\ FBS Fetal bovine serum \\ EGF Epidermal growth factor
}

\section{Introduction}

Metastatic prostate cancer is a leading cause of cancer morbidity and mortality. The skeleton is the principal organ for metastasis formation in prostate cancer, and bone metastases are frequently painful and debilitating. Whilst androgens are critical regulators of prostate carcinoma growth and progression, most patients respond only temporarily to androgen ablation therapy. Skeletal metastases are predominantly osteoblastic, as apparent both 
radiographically and histopathologically, and are associated with elevated serum level of bone-specific alkaline phosphatase, a marker of osteoblast proliferation [1, 2]. These observations suggest that the biological interaction between prostate carcinoma cells and osteoblasts contributes to the metastatic progression of prostate cancer.

In order to experimentally address how osteoblastic cells may influence prostate carcinoma cell biology upon formation of bone metastasis, the human, androgen-sensitive prostate carcinoma cell line LNCaP [3] was treated with culture medium conditioned by the human, osteoblastderived sarcoma cell line OHS [4], and subsequent multiplex profiling of LNCaP kinase activity was performed using flow-through microarrays with peptide substrates (Tyrosine Kinase PamChip ${ }^{\circledR}$ Arrays; PamGene International BV, 's-Hertogenbosch, The Netherlands), a novel platform that allows rapid, real-time measurements of phosphopeptide signatures generated by biological samples. As supplementary approaches, we used LNCaP cells in cocultures with either OHS cells or with osteoblastic cells that had been differentiated from human mesenchymal stem cells.

Insight into regulatory mechanisms underlying the establishment of prostate carcinoma cells within an osteoblastic microenvironment may eventually lead to more effective therapies to prevent or treat metastatic disease. Hence, from a therapeutic perspective, we compared the intracellular LNCaP signalling pathways activated by influence of osteoblastic cells with pathways induced by androgen treatment, to identify signalling networks potentially accessible for therapeutic targeting.

\section{Materials and methods}

\section{Cell cultures}

The LNCaP and OHS cell lines were routinely held in RPMI 1640 medium supplemented with $10 \%$ fetal bovine serum (FBS) and $2.0 \mathrm{mM}$ glutamine, defined as growth medium. Seventy-two hours before start of experimental incubations, monocultures of $\mathrm{LNCaP}$ and $\mathrm{OHS}$ were seeded in a total number of $1.0 \times 10^{6}$ cells in $75 \mathrm{~cm}^{2}$ cell flasks in RPMI containing 2\% charcoal-treated FBS and glutamine. After $48 \mathrm{~h}$, this medium was changed to RPMI containing $0.5 \%$ charcoal-treated FBS and glutamine, defined as experimental medium, for another $24 \mathrm{~h}$ before experimental incubations were started (at time 0 ). All cell cultures were invariably held in $10 \mathrm{ml}$ medium throughout different incubations.

At time 0 (start of the experimental incubations), LNCaP cells were refed with experimental medium supplemented with $100 \mathrm{nM}$ of the synthetic androgen analog
R1881 (methyltrienolone; Biocompare, Inc., South San Francisco, CA), giving rise to the $\mathrm{LNCaP}$ entity denoted androgenic, or with medium conditioned by OHS cells. The conditioned medium had been collected from OHS monocultures that had been grown for $48 \mathrm{~h}$ (relative to time 0) in experimental medium. This medium was subsequently diluted 1:10 in either fresh experimental medium or in medium obtained from standard $\mathrm{LNCaP}$ monocultures after $48 \mathrm{~h}$ of incubation (relative to time 0 ) in experimental medium, before the application onto monocultured $\mathrm{LNCaP}$ cells, giving rise to the $\mathrm{LNCaP}$ entities denoted paracrine 1 and paracrine 2 , respectively.

Cocultures of LNCaP/OHS were also seeded in a total number of $1.0 \times 10^{6}$ cells in $75 \mathrm{~cm}^{2}$ cell flasks in $10 \mathrm{ml}$ medium and were incubated identically to the monocultured cell lines prior to and during experimental incubations. Different ratios of LNCaP to OHS cells had been tested in a series of cocultures to find the optimal culturing conditions [5], and a 10:1 seeding ratio was chosen.

Differentiated mesenchymal stem cells from human bone marrow were prepared as previously described $[6,7]$. Briefly, bone marrow (10-20 ml) was aspirated from the posterior iliac crest of healthy, adult volunteers. Mononuclear cells were isolated by density gradient centrifugation and resuspended in complete medium, consisting of MEM $\alpha$ supplemented with $20 \%$ FBS and $2.0 \mathrm{mM}$ glutamine. After $24 \mathrm{~h}$, non-adherent cells were discarded, and the adherent cells were thoroughly washed and further cultured in complete medium for 7 days. Subsequently, the cells were detached and replated in osteogenic differentiation medium, consisting of MEM $\alpha$ with $10 \%$ FBS, $1.0 \mathrm{mM}$ glutamine, $10 \mathrm{nM}$ dexamethasone, $10 \mu \mathrm{M} \beta$-glycerol phosphate, and $200 \mu \mathrm{M}$ ascorbic acid. This medium was replaced every 3-4 days for about 20 days before LNCaP cells $\left(1.0 \times 10^{6}\right.$ cells in $75 \mathrm{~cm}^{2}$ cell flasks $)$ were seeded on top of the osteoblasts and the cocultures of $\mathrm{LNCaP}$ and differentiated stem cells were incubated identically to the other culture setups prior to and during experimental incubations.

In the experimental setups, $\mathrm{LNCaP}$ cells were harvested $48 \mathrm{~h}$ after time 0 , either directly from the various monocultures or after immunomagnetic cell segregation of cocultures (see below), and lysed in M-PER Mammalian Extraction Reagent containing Halt Phosphatase Inhibitor Cocktail and EDTA-free Halt Protease Inhibitor Cocktail (Pierce Biotechnology, Inc., Rockford, IL). Reference lysates were made from monocultured $\mathrm{LNCaP}$ grown for $48 \mathrm{~h}$ (relative to time 0 ) in experimental medium only.

In the experimental setup involving epidermal growth factor (EGF; Sigma-Aldrich Norway AS, Oslo, Norway), EGF was added to LNCaP cells in a final concentration of $50 \mathrm{ng} / \mathrm{ml}$. 
Kinase activity profiling

The kinase substrate array technology allows functional comparison of biological samples without prior knowledge of which activity pathways are influenced by the experimental conditions. The high-throughput format of the Tyrosine Kinase PamChip ${ }^{\circledR}$ Array is based on the use of a porous, three-dimensional aluminum-oxide material as solid support for the substrates. The sample lysates are actively pumped through the interconnected capillary pores of the arrays to allow contact with the reactive surface for enzymatic reaction with the peptide substrates. The phosphorylation kinetics are therefore rapid and can be completed within few minutes, allowing the generation of spot images to be followed in real-time.

Each array contains 144 peptide substrates, and these target peptides consist of 13 or 14 amino acids with sites for phosphorylation, mainly tyrosine, representing 100 different proteins. Reaction mixtures consisted of $\mathrm{Abl}$ Reaction Buffer (50 mM Tris- $\mathrm{HCl} \mathrm{pH} 7.5,10 \mathrm{mM} \mathrm{MgCl}_{2}$, $1 \mathrm{mM}$ EGTA, $2 \mathrm{mM}$ dithiothreitol, 0.01\% Brij 35; New England BioLabs, Inc., Ipswich, MA), $1 \mathrm{mg} / \mathrm{ml}$ bovine serum albumin, $100 \mu \mathrm{M}$ ATP, and $12.5 \mu \mathrm{g} / \mathrm{ml}$ of the monoclonal, FITC-conjugated anti-phosphotyrosine antibody (Exalpha Biologicals, Inc., Maynard, MA), added to each sample lysate, containing $2-5 \mu \mathrm{g}$ total protein. The arrays were blocked with $20 \mu \mathrm{g} / \mathrm{ml}$ bovine serum albumin and subsequently washed twice with Abl Reaction Buffer before the reaction mixtures were applied for initiation of enzymatic reactions. Spot images were recorded after each completed pumping cycle by a charge-coupled device camera until the reactions were terminated.

Raw and processed data and investigation and array designs can be obtained from the EMBL-EBI ArrayExpress database (www.ebi.ac.uk) by accession number E-TABM626.

Microarray data adaptation and statistical analysis

Six independent $\mathrm{LNCaP}$ reference samples (denoted LNCaP baseline), one of each $\mathrm{LNCaP}$ treated sample (paracrine 1, paracrine 2, androgenic), one sample each of LNCaP and OHS cells immunosegregated from coculture (see below), and two independent samples of monocultured OHS cells were analyzed, and each sample was measured in three independent reactions. The image information was converted using BioNavigator software (PamGene International BV, www.pamgene.com). For each spot on the array, signal intensity after background subtraction was calculated and used for further analysis. Data normalization of spot signal intensities and subsequent comparison analyses were conducted using GeneSpring software (Agilent Technologies, www.home.agilent.com). All values were normalized to the calculated mean value of all substrate phosphorylation intensities in the $\mathrm{LNCaP}$ baseline sample. In the $\mathrm{LNCaP}$ treated samples, the criterion for selecting a substrate with significantly increased phosphorylation level compared to the corresponding value in the baseline sample was set at a statistical difference of $P<0.05$ (Student's $t$-test). Using this criterion, the foldincrease $\left(\log _{2}\right)$ of substrate phosphorylation levels was within a range of 0.85 to $>5.2$.

Following the statistical analysis, two different pathway visualization systems were used to create information about pathway connectivity. Peptide names were generated from corresponding gene name entries and protein sequences in SwissProt (http://au.expasy.org/sprot) to enable unambiguous annotation of the peptide sequences and phosphorylation sites. It should be noted that, for some proteins, multiple peptides have been derived and are present on the Tyrosine Kinase PamChip ${ }^{\circledR}$ Array. For the pathway connectivity analysis, one list of peptide identifications for each of the treated samples was generated. First, those lists were loaded into PathwayArchitect software (Stratagene Corp., www.stratagene.com; Strand Life Sciences Pvt. Ltd., www.avadis.strandgenomics.com), and the program was used to generate a new list that contained all peptides in the others, but where duplicates had been removed. The peptide identifications in the final list were visualized through a direct interaction network, defined to show all interactions between peptides that were of the following types: binding, expression, protein modification, and regulation. Some peptides were omitted from the network because the interactions were below maximum score/quality, which is defined as an interaction from manually curated sources in the database. The direct interaction network was exported, all interactions were manually checked, and uninteresting interactions were removed. These manual edits were incorporated into the interaction network. Subsequently, the peptide list identified for each of the treated samples was imported into PathwayStudio software (Ariadne Genomics, www.ariadnegenomics.com), and pathways were created directly from the ResNet database (Ariadne Genomics), which comprises both curated and canonical pathways. The peptide set was cross-checked with these pathways, and a pathway list was generated and ranked by the hypergeometric probability factor. All interactions were manually inspected and selected on the criterion of the highest number of proteins being involved in a linear pathway or sub-pathway, by superposition of the peptide set to a cartoon representation of the pathway reported.

Western blot analysis

Expression of phosphoproteins was measured by means of standard western immunoblots. Primary, polyclonal 
antibodies, selected against the same phosphorylated epitopes as the corresponding target peptides on the microarrays, were anti-phospho(y1197)-EGFR (Abcam Plc., Cambridge, UK) and anti-phospho(y1248)-Neu (Santa Cruz Biotechnology, Inc., Santa Cruz, CA). Further, the monoclonal anti-EGFR antibody 425.3 [8], a polyclonal anti-ErbB2 antibody (R\&D Systems Europe Ltd., Abingdon, UK), and a monoclonal anti- $\alpha$-tubulin antibody (Calbiochem/Merck Biosciences Ltd., Nottingham, UK) were used.

Immunomagnetic cell separation

MOC-31 (IQ Corporation BV, Groningen, The Netherlands) is an IgG1 class antibody that binds to the EPCAM antigen, which is consistently expressed in most epithelial cells [9]. The high-affinity monoclonal antibody 9.2.27, which was originally developed against melanoma [10], recognizes an epitope on the high molecular weight melanoma-associated antigen and has also been shown to bind some subgroups of sarcoma, including osteosarcoma [11, 12]. The antibodies were conjugated to superparamagnetic particles coated with polyclonal sheep-antimouse IgG particles (Dynabeads; Dynal A.S., Oslo, Norway).

At the end of experimental incubations, cocultures of LNCaP cells with OHS cells and of LNCaP cells with differentiated mesenchymal stem cells were detached, and the resulting single cell suspensions were subjected to immunomagnetic target cell isolation, essentially as previously described [12-15]. The positive cell fractions were examined by light microscopy for the principal presence of cells with $\geq 5$ immunobeads bound to their surface (bead rosettes). For the purpose of cell quantification and quality assurance, the immunosegregation procedure was repeated with 9.2.27-coated beads added to the negative cell fractions for isolation of OHS cells. The segregated cell fractions from $\mathrm{LNCaP} / \mathrm{OHS}$ coculture samples $\left(10^{6}-10^{7}\right.$ cells) contained 60-70\% MOC-31-selected bead rosettes and $\sim 30 \%$ 9.2.27-selected bead rosettes.

\section{Results}

Kinase activity evoked by osteoblastic and androgenic influence

Monocultured LNCaP cells were treated with OHS-conditioned medium to experimentally replicate the biological context of paracrine osteoblastic influence. Moreover, to simulate the complex processes involved in aberrant activation of the androgen signalling axis in prostate cancer $[16,17]$, the experimental setup included LNCaP cells treated with the synthetic androgen analog R1881 to observe whether androgen receptor-mediated signalling pathways might differ from pathways activated by OHSdirected influence. Given the assumption that biologically relevant signalling events implicated in the metastatic phenotype require sustained activation, 48-h incubation times were used for the experimental LNCaP contexts.

From the tyrosine kinase microarray analysis, the substrate phosphorylation state generated by each LNCaP entity (paracrine, androgenic) was calculated with respect to baseline (untreated LNCaP cells). The identified substrates showed increases in phosphorylation level within a broad range (Table 1).

Each individual substrate phosphorylation signature was considered to represent a subset of the information flow through the globally activated signalling network of the particular LNCaP entity. Tools for analyzing interconnectivity of biological molecules have so far been used primarily to explore system-level gene expression data. However, such computational methods may also elucidate how kinase activity information is directed, and by using these algorithms, we assumed that phosphorylation events that appeared simultaneously might be interlinked and provide information about pathway connectivity [18, 19].

By applying these assumptions, the network interaction analysis omitted phosphorylated substrates that did not appear within any signalling pathway when defined by the interaction types delineated in "Materials and methods". As illustrated in Fig. 1, the resulting network connectivity map indicated that signalling pathways involved in cell adhesion and motility as well as cell proliferation were activated in the paracrine LNCaP entity. Activation of similar but also completely unrelated proliferation pathways was observed in the androgenic entity. Interestingly, only the signalling pathway mediated by EGFR seemed to be activated by the influence of both osteoblastic cells and androgen treatment.

Since the technology for multiplex kinase activity profiling and computational tools for data analysis are at an early stage of development [20], no general consensus approach to data validation exists. We therefore performed conventional western immunoblotting for selected, individual phosphoproteins expressed by LNCaP cells [21, 22] that in addition are representative of signalling pathways and therapeutic targets under investigation in recently conducted trials of metastatic prostate cancer [23-26]. Markedly increased expression levels of EGFR phosphorylated on tyrosine 1197 and ERBB2 phosphorylated on tyrosine 1248 were found in both the paracrine and androgenic LNCaP entity (Fig. 2a). In contrast, EGFR was found to be non-phosphorylated following $48 \mathrm{~h}$ of incubation with either EGF or $10 \%$ FBS, whereas shorttime EGF treatment caused transient increase in EGFR phosphorylation (Fig. 2b), which is in accordance with 
Table 1 Peptide substrates with increased phosphorylation levels generated by the paracrine and androgenic LNCaP entities

\begin{tabular}{|c|c|c|}
\hline Peptide substrate & Paracrine $^{\mathrm{a}}$ & Androgenic \\
\hline EGFR_y1197 ${ }^{\mathrm{b}}$ & $1.84^{\mathrm{c}}$ & 1.94 \\
\hline EGFR_y1110 & 2.34 & - \\
\hline RB1_s807/s811 & 3.39 & 3.07 \\
\hline ERBB2_y877 & 1.67 & - \\
\hline ERBB2_y1248 & 1.56 & - \\
\hline CDK2_t14/y15 & 1.86 & - \\
\hline EPHB1_y778 & 2.11 & - \\
\hline IRS2_y919 & 5.56 & - \\
\hline JAK1_y1022/y1023 & 2.05 & - \\
\hline LAT_y200 & 3.49 & - \\
\hline LCK_y394 & 1.91 & - \\
\hline MET_y1230/y1234/y1235 & 1.57 & - \\
\hline MST1R_y1353 & 2.86 & - \\
\hline MST1R_y1356/y1360 & 3.38 & - \\
\hline PDPK1_y9 & 1.82 & - \\
\hline PDPK1_y373/y376 & 1.82 & - \\
\hline PTK2_y576/y577 & 1.39 & - \\
\hline PTK2B_y579/y580 & 1.21 & - \\
\hline RASA1_y460 & 1.63 & - \\
\hline RET_y1029 & 1.76 & - \\
\hline ZAP70_y492/y493 & 1.93 & - \\
\hline CREB1_y134/s133 & - & 1.47 \\
\hline ERBB4_y1284 & - & 3.31 \\
\hline GSK3B_y216 & - & 1.15 \\
\hline RAF1_s337/s338/y339/y340 & - & 1.14 \\
\hline CHRNB1_y390 & 1.57 & 1.57 \\
\hline LTK_y772/y776/y777 & - & 1.20 \\
\hline DDR1_y792/y796/y797 & 1.20 & - \\
\hline MAPK10_t221/y223 & 1.32 & - \\
\hline MAPK12_t183/y185 & - & - \\
\hline PECAM1_y713 & 2.06 & - \\
\hline PRRX2_y214 & 1.48 & - \\
\hline ANXA1_y20/t23 & 2.41 & - \\
\hline CD79A_y182/y188 & 1.75 & - \\
\hline CTTN1_y477/y483 & 2.01 & - \\
\hline CTTN1_y499 & 2.13 & - \\
\hline ENO2_y43 & 1.85 & - \\
\hline EPHA2_y772 & 1.99 & - \\
\hline EPHA7_y608/y614 & 2.27 & - \\
\hline EPOR_y368 & 2.08 & - \\
\hline EPOR_y426 & 2.29 & - \\
\hline FER_y714 & 1.66 & - \\
\hline FES_y713 & 2.20 & - \\
\hline FGFR2_y769 & 1.77 & - \\
\hline FGFR3_y760 & 2.23 & - \\
\hline FRK_y387 & 2.02 & - \\
\hline LAT_y255 & 1.54 & - \\
\hline
\end{tabular}

Table 1 continued

\begin{tabular}{lll}
\hline Peptide substrate & Paracrine $^{\mathrm{a}}$ & Androgenic $^{-}$ \\
\hline NTRK2_y702/y706/y707 & 1.43 & - \\
PDGFRB_y579/y581 & 3.87 & - \\
PDGFRB_y716 & 1.78 & - \\
PIK3R1_y607/s608 & 2.09 & - \\
PXN_y31 & 1.88 & - \\
PXN_y118 & 2.07 & - \\
TEC_y519 & 1.68 & - \\
PFKFB1_s33 & - & 1.36 \\
PTPN11_y542 & - & 2.94 \\
SYN1_s9 & - & 1.20 \\
\hline
\end{tabular}

'-' denotes that the change in substrate phosphorylation level was not found to be significant $(P<0.05$, Student's $t$-test $)$

a Phosphopeptide values from the $\mathrm{LNCaP}$ paracrine 2 entity are given

${ }^{b}$ For each substrate, position of phosphorylation sites within the protein is indicated

${ }^{\mathrm{c}}$ Fold changes $\left(\log _{2}\right)$ relative to LNCaP baseline samples are listed

previously published data $[21,27,28]$ and may indicate that functional signalling in prostate carcinoma cells under influence of osteoblastic cells is diverse and complex [21].

EGFR/ERBB2 phosphorylation in supplementary

biological models

We used additional experimental setups as biological controls for the osteoblastic influence on prostate carcinoma cells; the first to model the direct interaction between $\mathrm{LNCaP}$ and OHS cells and the second to provide cells that might be more representative of physiological osteoblasts.

Culturing LNCaP cells with OHS cells caused substantial change in LNCaP morphology. The spindle-shaped feature of monocultured LNCaP cells (Fig. 3a) was rapidly lost upon direct contact with the OHS cells. In coculture, both cell types appeared rounded, although cytoplasmic processes were still apparent on LNCaP cells (Fig. 3b). Cellular morphology of the OHS cells (Fig. 3c), however, remained independent of the culturing conditions.

Non-hematopoietic stem cells in the bone marrow are capable of differentiating into a variety of tissue entities, including osteogenic cells of bone tissue [29]. Incubation of mononuclear cells isolated from adult, human bone marrow with mesenchymal stem cell-stimulating medium followed by osteogenic differentiation medium $[6,7]$ gave rise to cells with osteoblastic characteristics, for example mineral deposition (Fig. 3d, e) and alkaline phosphatasesecreting activity (not shown), to be used in coculture with LNCaP cells (Fig. 3f). 
Fig. 1 Interconnected signalling pathways activated in LNCaP cells by influence of osteoblastic cells or androgen treatment. Two pathway visualization systems were applied to the data set (Table 1), which resulted in almost identical network connectivity maps. The substrate annotations are derived from gene name entries in SwissProt. The lines connecting nodes represent interactions of the following types: binding, expression, protein modification, and regulation. Yellow and blue nodes symbolize substrates phosphorylated by the paracrine and androgenic $\mathrm{LNCaP}$ entities, respectively. (Color figure online)

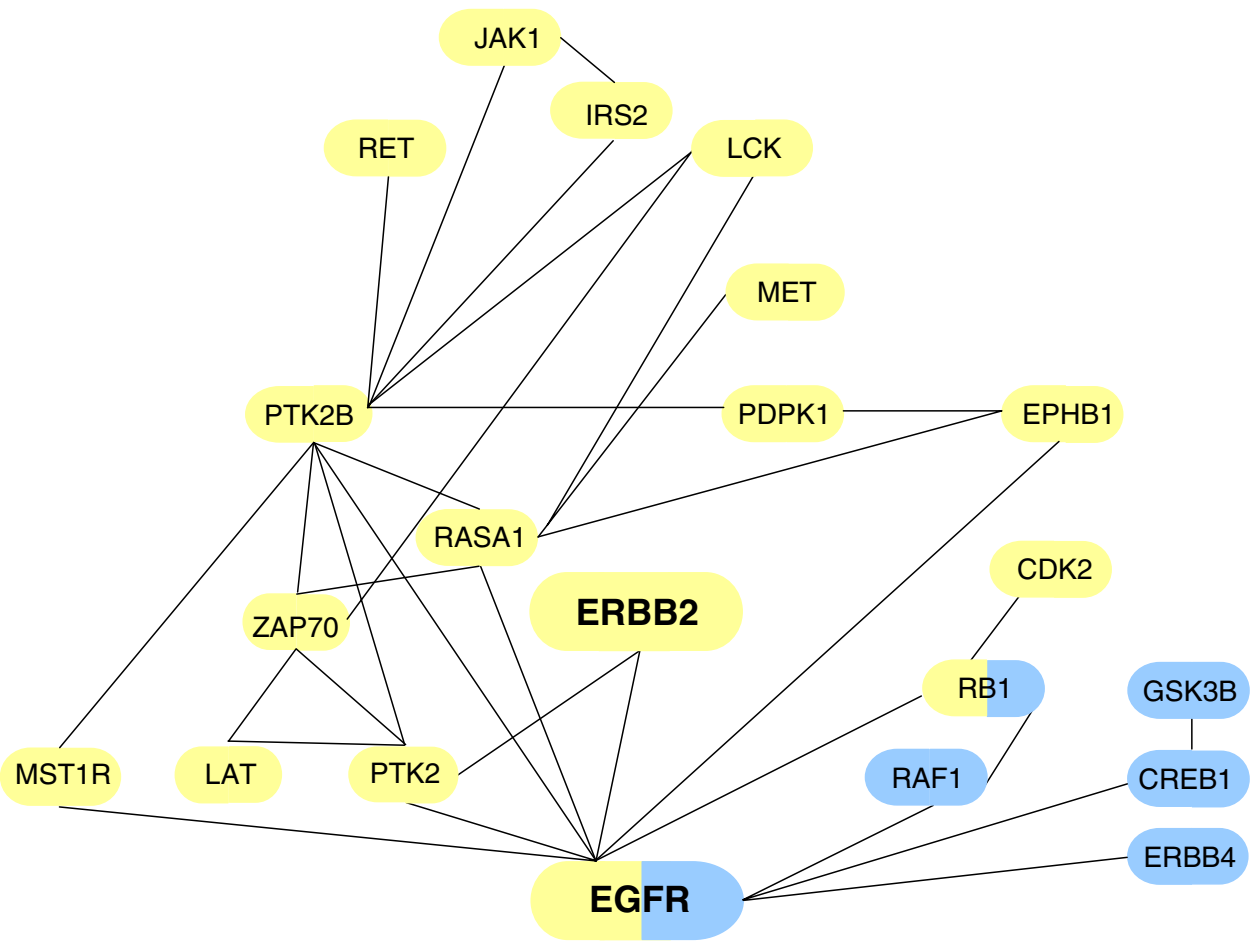

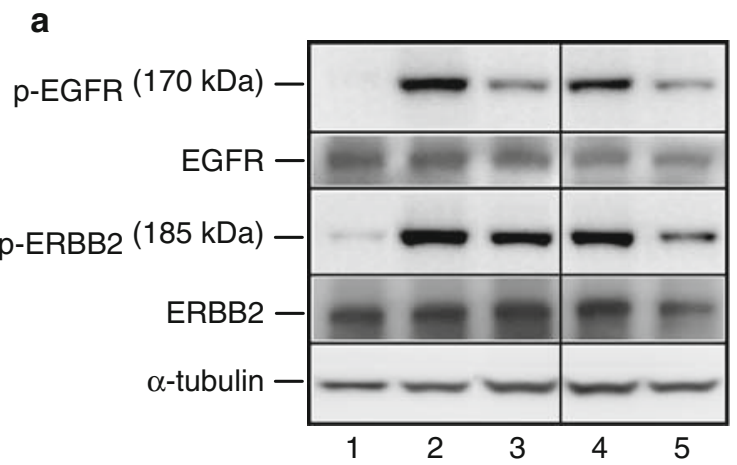

b

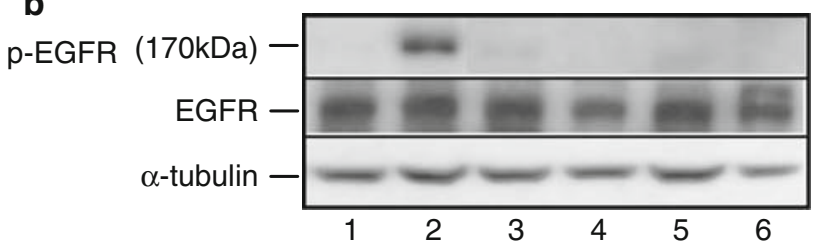

Fig. 2 Expression of phospho(y1197)-EGFR and phospho(y1248)ERBB2 in LNCaP cells. a Cells were harvested after $48 \mathrm{~h}$ of experimental treatments. Lanes: (1) baseline, (2) paracrine entity, (3) androgenic entity, (4) cells immunosegregated from coculture with OHS cells, and (5) cells immunosegregated from coculture with osteoblastic cells differentiated from human mesenchymal stem cells. b Cells were treated with $50 \mathrm{ng} / \mathrm{ml}$ EGF or $10 \%$ FBS. Lanes: (1) baseline, (2) EGF for $15 \mathrm{~min}$, (3) EGF for $24 \mathrm{~h}$, (4) EGF for $48 \mathrm{~h}$, (5) FBS for $48 \mathrm{~h}$, and (6) $48 \mathrm{~h}$ untreated

We applied the immunomagnetic cell separation method for selective isolation of $\mathrm{LNCaP}$ cells from the cocultured osteoblastic cells (Fig. 3g) and subjected the isolated carcinoma cells to western immunoblotting. Increased expression levels of EGFR phosphorylated on tyrosine 1197 and ERBB2 phosphorylated on tyrosine 1248 were found in $\mathrm{LNCaP}$ cells from coculture with both $\mathrm{OHS}$ cells and osteoblastic cells that had been differentiated from human mesenchymal stem cells (Fig. 2a).

\section{Supplementary kinase activity profiling}

To assess robustness of the biological models in the analytical application of multiplex kinase activity profiling, three experimental setups were exploited.

Kinase activity was compared in LNCaP cells treated with medium conditioned by OHS cells only and with the addition of medium obtained from standard LNCaP monocultures (LNCaP entities denoted paracrine 1 and paracrine 2 , respectively). In this experimental setting, the profile of phosphorylated substrates generated by the LNCaP cells did not significantly change by the addition of medium conditioned by LNCaP monocultures (compare Table 1 with Table 2), strongly indicating that the signalling pathways of biological importance in the paracrine setting were activated by factors secreted by the OHS cells exclusively.

Kinase activity induced in LNCaP cells by direct contact with OHS cells was analyzed following carcinoma cell isolation from cocultures. The substrate phosphorylation state generated by such $\mathrm{LNCaP}$ cells overlapped the phosphopeptide signatures identified in the paracrine LNCaP entities (Table 2). 

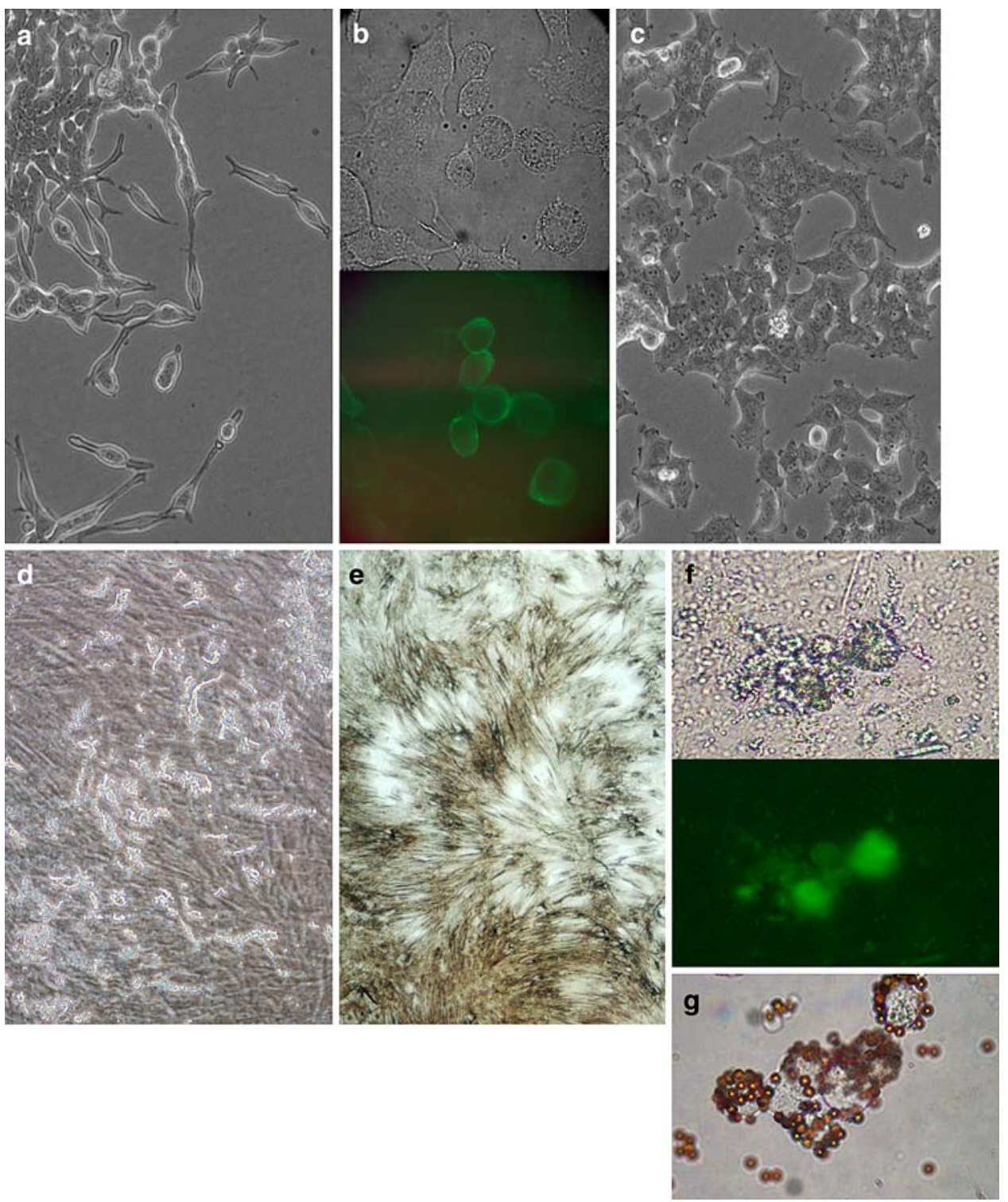

Fig. 3 Morphologic characteristics of $\mathrm{LNCaP}$ and osteoblastic cells. a Monocultured LNCaP cells, untreated (magnification $\times 300$ ). b $\mathrm{LNCaP} / \mathrm{OHS}$ coculture, at start of experimental incubation (magnification $\times 400$ ). The fluorescent cells are stained with FITCconjugated 9.2.27 antibody; accordingly, representing OHS cells. c Monocultured OHS cells (magnification $\times 300$ ). d Mononuclear cells isolated from adult human bone marrow were cultured in mesencymal stem cell-stimulating medium followed by osteogenic differentiation medium for 20 days to obtain in vitro-differentiated osteoblasts (magnification $\times 300$ ). e The differentiated osteoblasts were fixed in ice-cold methanol for $30 \mathrm{~min}$, incubated in dark with 5\% silver nitrate solution for $30 \mathrm{~min}$, and washed thoroughly with deionized water and then with $5 \% \mathrm{Na}_{2} \mathrm{CO}_{3}$ and $0.2 \%$ formaldehyde, according to the von Kossa staining protocol for calcium salts. Mineral deposits are visualized as dark brown stain (magnification $\times 300$ ). $\mathbf{f}$ Coculture of LNCaP cells and in vitro-differentiated osteoblasts, at start of experimental incubation (magnification $\times 400$ ). The fluorescent cells are stained with FITC-conjugated MOC-31 antibody; accordingly, representing LNCaP cells. $\mathrm{g}$ Suspended cells binding MOC-31-coated beads; accordingly, representing LNCaP cells, after immunomagnetic selection from LNCaP/OHS coculture (magnification $\times 400$ ) (Color figure online)
We have previously evaluated the immunomagnetic cell separation method for selective isolation of target cells and demonstrated that target cell populations are highly enriched [12-15]. But because kinase activity profiling might be sensitive to the possible presence of contaminants, we screened for phosphopeptide signatures generated by immunoselected as well as monocultured OHS cells. Notably, the resulting substrate phosphorylation patterns were closely similar for the two conditions (Table 3) and also clearly distinguishable from those generated by the $\mathrm{LNCaP}$ entities, which argues against significant contamination of OHS cells in MOC-31-positive LNCaP cell isolates. 
Table 2 Peptide substrates with increased phosphorylation levels generated by the LNCaP paracrine 1 entity and $\mathrm{LNCaP}$ cells immunosegregated from coculture with OHS cells (co-LNCaP)

\begin{tabular}{|c|c|c|}
\hline Peptide substrate & Paracrine 1 & Co-LNCaP \\
\hline EGFR_y1197 ${ }^{\mathrm{a}}$ & $1.50^{\mathrm{b}}$ & 2.16 \\
\hline EGFR_y1110 & - & 1.86 \\
\hline RB1_s807/s811 & 3.72 & - \\
\hline ERBB2_y877 & 1.06 & - \\
\hline ERBB2_y1248 & - & - \\
\hline CDK2_t14/y15 & 1.47 & - \\
\hline EPHB1_y778 & 1.78 & - \\
\hline IRS2_y919 & 4.88 & - \\
\hline JAK1_y1022/y1023 & 1.81 & - \\
\hline LAT_y200 & 2.70 & 2.94 \\
\hline LCK_y394 & 1.38 & - \\
\hline MET_y1230/y1234/y1235 & 1.08 & - \\
\hline MST1R_y1353 & 2.29 & 2.20 \\
\hline MST1R_y1356/y1360 & 2.60 & 2.01 \\
\hline PDPK1_y9 & 1.50 & - \\
\hline PDPK1_y373/y376 & 1.50 & - \\
\hline PTK2_y576/y577 & 1.33 & 1.03 \\
\hline PTK2B_y579/y580 & 0.85 & 0.85 \\
\hline RASA1_y460 & 1.27 & 0.96 \\
\hline RET_y1029 & 1.24 & - \\
\hline ZAP70_y492/y493 & 1.68 & 1.21 \\
\hline CREB1_y134/s133 & - & - \\
\hline ERBB4_y1284 & - & - \\
\hline GSK3B_y216 & - & - \\
\hline RAF1_s337/s338/y339/y340 & - & - \\
\hline CHRNB1_y390 & 1.00 & 0.95 \\
\hline LTK_y772/y776/y777 & - & 0.96 \\
\hline DDR1_y792/y796/y797 & 1.11 & 0.91 \\
\hline MAPK10_t221/y223 & 1.28 & 0.98 \\
\hline MAPK12_t183/y185 & - & 2.23 \\
\hline PECAM1_y713 & 1.51 & 0.87 \\
\hline PRRX2_y214 & 1.23 & 0.93 \\
\hline ANXA1_y20/t23 & 1.93 & - \\
\hline CD79A_y182/y188 & 1.39 & - \\
\hline CTTN1_y477/y483 & 1.45 & - \\
\hline CTTN1_y499 & 1.64 & - \\
\hline ENO2_y43 & 1.43 & - \\
\hline EPHA2_y772 & 1.60 & - \\
\hline EPHA7_y608/y614 & 1.64 & - \\
\hline EPOR_y368 & 1.67 & - \\
\hline EPOR_y426 & 1.77 & - \\
\hline FER_y714 & 1.36 & - \\
\hline FES_y713 & 1.82 & - \\
\hline FGFR2_y769 & 1.28 & - \\
\hline FGFR3_y760 & 1.63 & - \\
\hline FRK_y387 & 1.47 & - \\
\hline LAT_y255 & 1.13 & - \\
\hline
\end{tabular}

Table 2 continued

\begin{tabular}{lll}
\hline Peptide substrate & Paracrine 1 & Co-LNCaP \\
\hline NTRK2_y702/y706/y707 & 1.08 & - \\
PDGFRB_y579/y581 & 3.52 & - \\
PDGFRB_y716 & 1.59 & - \\
PIK3R1_y607/s608 & 1.59 & - \\
PXN_y31 & 1.44 & - \\
PXN_y118 & 1.61 & - \\
TEC_y519 & 1.23 & - \\
PFKFB1_s33 & - & - \\
PTPN11_y542 & - & - \\
SYN1_s9 & - & - \\
\hline
\end{tabular}

'-' denotes that the change in substrate phosphorylation level was not found to be significant $(P<0.05$, Student's $t$-test $)$

${ }^{a}$ For each substrate, position of phosphorylation sites within the protein is indicated

${ }^{\mathrm{b}}$ Fold changes $\left(\log _{2}\right)$ relative to LNCaP baseline samples are listed

\section{Discussion}

Given the considerable health care challenges generated by the prevalence of metastatic bone disease in prostate cancer, the apparent shortage of adequate experimental models to study this aspect of disease progression is striking. From a clinical point of view, prostate cancer metastasis to bone is a lengthy and complex disease process, which makes it difficult to establish adequate experimental models to recreate all steps involved. As highlighted in a comprehensive review [30], most of the experimental systems examining this phenomenon are based on rodent models. Importantly, the model systems used in this study exclusively utilize cell types of human origin, but caution should be exercised regarding any compelling conclusion based on specific in vitro experimental conditions, which can not reflect an intact microenvironmental in vivo setting. Nevertheless, given that regulatory mechanisms implicated in the metastatic phenotype are evoked when carcinoma cells settle within an osteoblastic microenvironment, the combination of our experimental models and analytical technology may provide relevant information about functional signalling networks that facilitate this biological process, and in addition may enable the identification of new targets for therapeutic intervention.

The OHS cell line was originally established from a patient with aggressive osteosarcoma [4], and it might be argued that it is not representative for physiological osteoblasts. However, formation of osteosclerotic (i.e., osteoblastic) lesions following intratibial OHS cell inoculation has previously been demonstrated by radiographic, scintigraphic, and morphologic assessments [31]. 
Table 3 Phosphopeptides generated by monocultured OHS cells (mono-OHS) and OHS cells immunosegregated from coculture with LNCaP cells (co-OHS)

\begin{tabular}{|c|c|c|}
\hline Peptide substrates & Mono-OHS & $\mathrm{Co}-\mathrm{OHS}$ \\
\hline ANXA1_y20/t23 ${ }^{\mathrm{a}}$ & $-1.56^{\mathrm{b}}$ & -2.84 \\
\hline CD79A_y182/y188 & -3.70 & -4.54 \\
\hline CDK2_t14/y15 & -0.94 & -1.32 \\
\hline CHRNB1_y390 & -0.49 & -0.64 \\
\hline CREB1_y134/s133 & -0.81 & -1.29 \\
\hline CTTN1_y477/y483 & -0.86 & -1.43 \\
\hline CTTN1_y499 & -1.56 & -2.00 \\
\hline DCX_y112/s116 & -0.92 & -0.86 \\
\hline DDR1_y792/y796/y797 & -0.40 & -0.79 \\
\hline EFS_y253 & -0.86 & -1.18 \\
\hline ENO2_y43 & -3.72 & -4.76 \\
\hline EPHA2_y772 & -1.43 & -2.32 \\
\hline EPHA7_y608/y614 & -1.60 & -2.18 \\
\hline EPHB1_y778 & -1.60 & -2.40 \\
\hline EPOR_y368 & -0.94 & -1.40 \\
\hline EPOR_y426 & -0.94 & -2.32 \\
\hline ERBB2_y877 & -1.74 & -2.18 \\
\hline ERBB2_y1248 & -1.22 & -2.06 \\
\hline FER_y714 & -1.15 & -1.64 \\
\hline FES_y713 & -1.03 & -2.25 \\
\hline FGFR2_y769 & -1.89 & -2.06 \\
\hline FGFR3_y760 & -1.56 & -0.89 \\
\hline FRK_y387 & -1.22 & -1.84 \\
\hline GSK3B_y216 & -1.25 & -0.97 \\
\hline JAK1_y1022/y1023 & -2.18 & -2.32 \\
\hline KRT6E_s59 & -0.92 & -1.47 \\
\hline LAT_y255 & -1.29 & -2.40 \\
\hline LCK_y394 & -1.60 & -2.25 \\
\hline LTK_y772/y776/y777 & -0.86 & -1.03 \\
\hline MAPK10_t221/y223 & -0.94 & -0.62 \\
\hline MET_y1230/y1234/y1235 & -0.89 & -1.12 \\
\hline NCF1_s315/s320 & -0.67 & -0.74 \\
\hline NTRK1_y496 & -1.94 & -2.84 \\
\hline NTRK2_y702/y706/y707 & -2.94 & -3.84 \\
\hline PDGFRB_y579/y581 & -4.32 & -3.84 \\
\hline PDGFRB_y716 & -1.51 & -1.00 \\
\hline PDGFRB_y771/y775/y778 & -1.00 & -1.25 \\
\hline PDPK1_y9 & -1.40 & -1.25 \\
\hline PDPK1_y373/y376 & -1.36 & -2.00 \\
\hline PECAM1_y713 & -1.06 & -1.47 \\
\hline PFKFB1_s33 & -0.79 & -0.92 \\
\hline PIK3R1_y607/s608 & -1.25 & -2.56 \\
\hline PLCG1_y771 & -4.57 & -4.64 \\
\hline PRRX2_y214 & -1.36 & -1.25 \\
\hline PTK2_y576/y577 & -1.15 & -0.81 \\
\hline PTK2B_y579/y580 & -0.71 & -1.51 \\
\hline PXN_y31 & -1.25 & -1.84 \\
\hline
\end{tabular}

Table 3 continued

\begin{tabular}{llc}
\hline Peptide substrates & Mono-OHS & Co-OHS \\
\hline PXN_y118 & -1.03 & -1.29 \\
RAF1_s337/s338/y339/y340 & -0.89 & -1.18 \\
RASA1_y460 & -1.36 & -1.09 \\
RET_y1029 & -1.22 & -2.18 \\
SYN1_s9 & -1.06 & -1.40 \\
TEC_y519 & -1.51 & -1.64 \\
TYRO3_y686 & -1.40 & -1.29 \\
ZAP70_y492/y493 & -1.56 & -1.74 \\
\hline a For each substrate, position of phosphorylation sites & within the \\
protein is indicated & & \\
b Fold changes $\left(\log _{2}\right)$ relative to LNCaP baseline sample are listed
\end{tabular}

Kinase activity microarrays represent an emerging technology that may become a powerful tool for signal transduction profiling of biological samples. A key advantage of this technology lies in its ability to provide signalling pathway maps that indicate the state of information flow through intracellular networks. Essentially, the peptide substrate array technology determines kinase activity profiles, which is different from but complementary to mass spectrometry, which identifies phosphorylated proteins that represent the end products of kinase activity. An additional advantage of kinase activity microarrays is their robustness with small sample quantities, typically 2 $5 \mu \mathrm{g}$ total protein being sufficient for Tyrosine Kinase PamChip ${ }^{\circledR}$ Array analysis, in contrast to several milligrams of total protein usually required for mass spectrometry analysis.

Of note, the phosphopeptide signatures generated by the LNCaP paracrine 1 and paracrine 2 entities and by $\mathrm{OHS}$ cells cultured alone or with $\mathrm{LNCaP}$ cells, respectively, were essentially identical. This indicates low variation between biologically similar samples and a high degree of reproducibility using this peptide substrate array technology.

Computational methods for mapping of phosphorylation networks are being developed, and reconstruction of EGFR-mediated pathways have been used to test applicability of such models [32]. Correct network definition may be restricted by the influence of contextual factors, such as subcellular compartmentalization or temporal expression of the proteins involved [33]. Therefore, to predict pathway connectivity with sufficient accuracy, the use of more than one computational technique on the dataset of interest has been recommended [20]. In this study, two different pathway visualization systems identified signalling networks that were essentially identical.

The signalling pathway mediated by EGFR was found to be activated in androgen-sensitive LNCaP cells under the influence of either osteoblastic cells or androgen treatment. 
Analysis by conventional western immunoblotting also revealed that ERBB2-dependent signalling was activated in both the paracrine and androgenic LNCaP entities, although the latter did not seem to generate this particular substrate phosphorylation profile on the microarray analysis. The kinase activity and protein expression data together strongly indicates a central involvement of both signalling pathways in the interaction between prostate carcinoma cells and osteoblasts.

Additionally, given that EGFR and ERBB2 are phosphorylated in androgen-sensitive LNCaP cells upon influence of osteoblasts, a functional androgen signalling axis $[16,17]$ may appear to be permissive for activity of these particular pathways in prostate cancer. Not the less, EGFR phosphorylation resulting from short-term incubation with bone stromal conditioned medium (containing $6 \%$ FBS) and suppression of in vivo bone metastasis formation following targeted inhibition of EGFR-dependent signalling have been demonstrated in androgen-independent prostate carcinoma PC3 cells [34]. Separately, EGFR and ERBB2 may facilitate androgen receptor-driven activity in prostate cancer at the level of target gene transcription in the absence or at low concentrations of androgens [35, 36]. Yet, androgen receptor pathway genes, identified by systemlevel analysis of gene expression in primary tumor specimens from therapy-naïve prostate cancer patients, were reported to be down-regulated, with a few exceptions, in lymph-node metastases from the patients [37]. This finding further supports the assumption that the regulatory control by the androgen receptor on carcinoma cell biology is lost in the process of prostate cancer metastasis, even in the presence of activating receptor ligands.

Of importance, our experimental data suggests that targeted inhibition of the signalling pathways directed by EGFR or ERBB2 may simultaneously ablate androgendriven proliferation of prostate carcinoma cells and the survival responses within an osteoblastic microenvironment. It equally provides a biological rationale for the use of EGFR or ERBB2 inhibition in systemic prevention or treatment of metastatic prostate cancer in the androgensensitive stage of the disease. Intriguingly, the therapeutic concept of EGFR or ERBB2 inhibition in hormonerefractory prostate cancer has recently been evaluated; however, in initial studies addressing the use of singleagent therapies in patients with androgen-resistant disease, neither receptor-blocking antibodies nor small-molecular tyrosine kinase inhibitors showed clinically significant activity [23-26].

Of note, the tumor suppressor PTEN phosphatase is frequently found to be functionally inactivated in prostate cancer, leading to increased activity of AKT kinase signalling independent of the up-stream EGFR/ERBB2 and to insensitivity to EGFR/ERBB2 inhibitors [16, 38-40]. The
LNCaP cells are deficient in PTEN [41, 42], questioning the relevance of extrapolating from our data that EGFR/ERBB2 signalling is involved in the maintenance of osteoblastinduced survival responses in prostate cancer. On the other hand, development of androgen independence and more advanced disease is associated with increased PTEN loss [43-45], favoring therapeutic EGFR/ERBB2 inhibition in the initial, androgen-sensitive stage of the disease. Thus, if exploitable in patients with therapy-naïve prostate cancer, inhibitory EGFR/ERBB2 targeting might be incorporated into treatment schedules with a potential reduction in the alternative requirement of long-term androgen depletion, a reduction in related side effects, and, intriguingly, the potential for an improvement in patient survival.

Acknowledgments We thank Dr. R. Reisfeld for the gift of the 425.3 and 9.2.27 antibodies. We are grateful to Ingrid J. Guldvik and Heidi Rasmussen for technical assistance. This study was supported by The Norwegian Cancer Society grant C-04083 and grants from Faculty Division The Norwegian Radium Hospital (to A. H. Ree). Bioinformatics service was provided by the Norwegian Microarray Consortium at the national technology platform (http://microarray.no ), supported by the Functional Genomics Program of the Norwegian Research Council.

Conflict of interest statements A. H. Ree, ̊. Bratland, P. J. Boender, and R. Ruijtenbeek are owners of a patent for diagnostic application of the Tyrosine Kinase PamChip ${ }^{\circledR}$ Array technology in prostate cancer (WIPO no. WO/2008/125633). P. J. Boender and R. Ruijtenbeek are employees of PamGene International BV.

Open Access This article is distributed under the terms of the Creative Commons Attribution Noncommercial License which permits any noncommercial use, distribution, and reproduction in any medium, provided the original author(s) and source are credited.

\section{References}

1. Roodman GD (2004) Mechanisms of bone metastasis. N Engl J Med 350:1655-1664. doi:10.1056/NEJMra030831

2. Logothetis CJ, Lin SH (2005) Osteoblasts in prostate cancer metastasis to bone. Nat Rev Cancer 5:21-28. doi:10.1038/nrc1528

3. Horoszewicz JS, Leong SS, Kawinski E et al (1983) LNCaP model of human prostatic carcinoma. Cancer Res 43:1809-1818

4. Fodstad Ø, Brøgger A, Bruland Ø et al (1986) Characteristics of a cell line established from a patient with multiple osteosarcoma, appearing 13 years after treatment for bilateral retinoblastoma. Int J Cancer 38:33-40. doi:10.1002/ijc.2910380107

5. Bratland ̊, Ragnhildstveit E, Bjørnland K et al (2003) The metalloproteinase inhibitor TIMP-2 is down-regulated by androgens in LNCaP prostate carcinoma cells. Clin Exp Metastasis 20:541-547. doi:10.1023/A:1025860214891

6. Colter DC, Class R, DiGirolamo CM et al (2000) Rapid expansion of recycling stem cells in cultures of plastic-adherent cells from human bone marrow. Proc Natl Acad Sci USA 97:32133218. doi:10.1073/pnas.070034097

7. Peister A, Mellad JA, Wang M et al (2004) Stable transfection of MSCs by electroporation. Gene Ther 11:224-228. doi:10.1038/ sj.gt.3302163 
8. Mueller BM, Romerdahl CA, Trent JM et al (1991) Suppression of spontaneous melanoma metastasis in scid mice with an antibody to the epidermal growth factor receptor. Cancer Res 51: 2193-2198

9. de Jonge MW, Kosterink JG, Bin YY et al (1993) Radioimmunodetection of human small cells lung carcinoma xenografts in the nude rat using ${ }^{111}$ in-labelled monoclonal antibody MOC31. Eur J Cancer 29A:1885-1890. doi:10.1016/0959-8049(93) 90543-O

10. Morgan AC Jr, Galloway DR, Reisfeld RA (1981) Production and characterization of a monoclonal antibody to a melanoma specific glycoprotein. Hybridoma 1:27-36

11. Godal A, Bruland OS, Haug E et al (1986) Unexpected expression of the $250 \mathrm{kD}$ melanoma-associated antigen in human sarcoma cells. Br J Cancer 53:839-841

12. Bruland OS, Hoifodt H, Saeter G et al (2005) Hematogenous micrometastases in osteosarcoma patients. Clin Cancer Res 11:4666-4673. doi:10.1158/1078-0432.CCR-05-0165

13. Forus A, Høifødt HK, Øverli GET et al (1999) Sensitive fluorescent in situ hybridisation method for the characterisation of breast cancer cells in bone marrow aspirates. J Clin Pathol Mol Pathol 52:68-74

14. Fodstad $\varnothing$, Faye R, Høifødt HK et al (2001) Immunobead-based detection and characterization of circulating tumor cells in melanoma patients. Recent Results in Cancer Res 158:40-50

15. Tveito S, Maelandsmo GM, Hoifodt HK et al (2007) Specific isolation of disseminated cancer cells: a new method permitting sensitive detection of target molecules of diagnostic and therapeutic value. Clin Exp Metastasis 24:317-327. doi:10.1007/s10585006-9052-8

16. Scher HI, Sawyers CL (2005) Biology of progressive, castrationresistant prostate cancer: directed therapies targeting the androgen-receptor signaling axis. J Clin Oncol 32:8253-8261. doi: 10.1200/JCO.2005.03.4777

17. Attard G, Sarker D, Reid A et al (2006) Improving the outcome of patients with castration-resistant prostate cancer through rational drug development. Br J Cancer 95:767-774. doi:10.1038/sj.bjc. 6603223

18. Petricoin EFIII, Bichsel VE, Calvert VS et al (2005) Mapping molecular networks using proteomics: a vision for patient-tailored combination therapy. J Clin Oncol 23:3614-3621. doi: 10.1200/JCO.2005.02.509

19. Sevecka M, MacBeath G (2006) State-based discovery: a multidimensional screen for small-molecule modulators of EGF signaling. Nat Methods 3:825-831. doi:10.1038/nmeth931

20. Janes KA, Lauffenburger DA (2006) A biological approach to computational models of proteomic networks. Curr Opin Chem Biol 10:73-80. doi:10.1016/j.cbpa.2005.12.016

21. Angelucci A, Festuccia C, Gravina GL et al (2004) Osteopontin enhances the cell proliferation induced by the epidermal growth factor in human prostate cancer cells. Prostate 59:157-166. doi: 10.1002/pros.20008

22. Berger R, Lin DI, Nieto M et al (2006) Androgen-dependent regulation of Her-2/neu in prostate cancer cells. Cancer Res 66:5723-5728. doi:10.1158/0008-5472.CAN-05-3928

23. de Bono JS, Bellmunt J, Attard G et al (2007) Open-label phase II study evaluating the efficacy and safety of two doses of pertuzumab in castrate chemotherapy-naïve patients with hormonerefractory prostate cancer. J Clin Oncol 25:257-262. doi:10.1200/ JCO.2006.07.0888

24. Agus DB, Sweeney CJ, Morris MJ et al (2007) Efficacy and safety of single-agent pertuzumab (rhuMAb $2 \mathrm{C} 4$ ), a human epidermal growth factor dimerization inhibitor, in castration-resistant prostate cancer after progression from taxane-based therapy. J Clin Oncol 25:675-681. doi:10.1200/JCO.2006.07.0649
25. Canil CM, Moore MJ, Winquist E et al (2005) Randomized phase II study of two doses of gefitinib in hormone-refractory prostate cancer: a trial of the National Cancer Institute of Canada-Clinical Trials Group. J Clin Oncol 23:455-460. doi:10.1200/JCO.2005. 02.129

26. Ziada A, Barqawi A, Glode LM et al (2004) The use of trastuzumab in the treatment of hormone refractory prostate cancer; phase II trial. Prostate 60:332-337. doi:10.1002/pros.20065

27. El Sheikh SS, Domin J, Abel P et al (2004) Phosphorylation of both EGFR and ErbB2 is a reliable predictor of prostate cancer cell proliferation in response to EGF. Neoplasia (New York, NY) 6:846-853. doi:10.1593/neo.04379

28. Martín-Orozco RM, Almaraz-Pro C, Rodríguez-Ubreva FJ et al (2007) EGF prevents the neuroendocrine differentiation of LNCaP cells induced by serum deprivation: the modulator role of PI3 K/Akt. Neoplasia (New York, NY) 9:614-624. doi:10.1593/ neo.07337

29. Giordano A, Galderisi U, Marino IR (2007) From the laboratory bench to the patient's bedside: an update on clinical trials with mesenchymal stem cells. J Cell Physiol 211:27-35. doi:10.1002/ jcp.20959

30. Singh AS, Figg WD (2005) In vivo models of prostate cancer metastasis to bone. J Urol 174:820-826. doi:10.1097/01.ju.0000 169133.82167.aa

31. Kjønniksen I, Winderen M, Bruland $\varnothing$ et al (1994) Validity and usefulness of human tumor models established by intratibial cell inoculation in nude rats. Cancer Res 54:1715-1719

32. Oda K, Matsuoka Y, Funahashi A et al. (2005) A comprehensive pathway map of epidermal growth factor receptor signaling. Mol Syst Biol 1:2005.0010

33. Linding R, Jensen LJ, Ostheimer GJ et al (2007) Systematic discovery of in vivo phosphorylation networks. Cell 129:14151426. doi:10.1016/j.cell.2007.05.052

34. Angelucci A, Gravina GL, Rucci N et al (2006) Suppression of EGF-R signaling reduces the incidence of prostate cancer metastasis in nude mice. Endocr Relat Cancer 13:197-210. doi: 10.1677/erc. 1.01100

35. Gregory CW, Fei X, Ponguta LA et al (2004) Epidermal growth factor increases coactivation of the androgen receptor in recurrent prostate cancer. J Biol Chem 279:7119-7130. doi:10.1074/jbc. M307649200

36. Mellinghoff IK, Vivanco I, Kwon A et al (2004) HER2/neu kinase-dependent modulation of androgen receptor function through effects on DNA binding and stability. Cancer Cell 6:517527. doi:10.1016/j.ccr.2004.09.031

37. Hendriksen PJ, Dits NF, Kokame K et al (2006) Evolution of the androgen receptor pathway during progression of prostate cancer. Cancer Res 66:5012-5020. doi:10.1158/0008-5472.CAN-05-3082

38. Feldman BJ, Feldman D (2001) The development of androgenindependent prostate cancer. Nat Rev Cancer 1:34-45. doi: $10.1038 / 35094009$

39. Bianco R, Shin I, Ritter CA et al (2003) Loss of PTEN/MMAC1/ TEP in EGF receptor-expressing tumor cells counteracts the antitumor action of EGFR tyrosine kinase inhibitors. Oncogene 22:2812-2822. doi:10.1038/sj.onc. 1206388

40. Wu Z, Gioeli D, Conaway M et al (2008) Restoration of PTEN expression alters the sensitivity of prostate cancer cells to EGFR inhibitors. Prostate 68:935-944. doi:10.1002/pros.20745

41. Li J, Yen C, Liaw D et al (1997) PTEN, a putative protein tyrosine phosphatase gene mutated in human brain, breast, and prostate cancer. Science 275:1943-1947. doi:10.1126/science.275.5308. 1943

42. Vlietstra RJ, van Alewijk DC, Hermans KG et al (1998) Frequent inactivation of PTEN in prostate cancer cell lines and xenografts. Cancer Res 58:2720-2723 
43. Rubin MA, Gerstein A, Reid K et al (2000) 10q23.3 loss of heterozygosity is higher in lymph node-positive $(\mathrm{pT} 2-3, \mathrm{~N}+)$ versus lymph node-negative (pT2-3,N0) prostate cancer. Hum Pathol 31:504-508. doi:10.1053/hp.2000.6713

44. Jiao J, Wang S, Qiao R et al (2007) Murine cell lines derived from Pten null prostate cancer show the critical role of PTEN in hormone refractory prostate cancer development. Cancer Res 67:6083-6091. doi:10.1158/0008-5472.CAN-06-4202

45. McCall P, Witton CJ, Grimsley S et al (2008) Is PTEN loss associated with clinical outcome measures in human prostate cancer? Br J Cancer 99:1296-1301. doi:10.1038/sj.bjc.6604680 